\title{
Service Quality Evaluation Method of City Shared Bicycles Based on SERVQUAL Model
}

\author{
Wang Shengjin, Wang Jichuan* \\ Shanghai Urban Construction Vocational College, Shanghai, 201415, China
}

\begin{abstract}
Bicycle sharing is an effective way to solve the problem of residents' last mile travel. Based on the SERVQUAL model. Build a service quality evaluation index system and evaluation model for shared bicycles. Taking Harbin's bicycle as an example, the quantitative evaluation of the service quality of shared bicycles was carried out, and the existing problems of the service quality of shared bicycles were identified and targeted improvement strategies were proposed. The research results can provide a scientific basis for improving the quality of city shared bicycle services.
\end{abstract}

\section{Introduction}

Bike sharing is an important part of the urban traffic microcirculation. As the new crown epidemic has not been completely eliminated, as major cities resume work and production, shared bicycles have become the first choice for many people to travel short distances. However, shared bicycles still have problems such as random parking, easy damage to the bicycles, and untimely dispatching. As a result, the service quality of shared bicycles needs to be improved. Therefore, it is necessary to adopt scientific methods to quantitatively evaluate the quality of urban shared bicycle service and propose improvement strategies.

\section{Evaluation index system of city shared bicycle service quality}

According to the content of the SERVQUAL service quality evaluation theory, the urban bike-sharing service has five attributes in the evaluation model: tangibility, reliability, responsiveness, security, and empathy. A brief adjustment was made to ensure that the five dimensions of the city's shared bicycle service quality remain unchanged, while adding multiple indicators to form a total of 27 specific problem items.

\subsection{Tangibility}

Tangibility refers to the facilities, equipment and staffing capabilities provided by the service. Seven indicators are set here: Whether the software platform is easy to operate; Whether the vehicle is comfortable and light to ride; Does the operating company have any measures to address the legal requirements of not being able to ride a bicycle under the age of 12; Whether the vehicle design is novel, fashionable, and Beautiful; The QR code is clear for damage; The condition of the vehicle is damaged; Whether the brake is sensitive during riding, and whether it can stop immediately.

\subsection{Reliability}

Reliability refers to the ability to perform service promises reliably and accurately. Here are 4 indicators: Whether activities such as credit free car rental are honoured; if the user encounters a sudden failure on the way, whether there is a rescue mechanism; whether the vehicle scheduling is timely and whether the car can be available at any time; whether the cycling records and deductions are accurate.

\subsection{Responsiveness}

Responsiveness refers to the willingness to provide prompt assistance to customers and improve service levels. Five indicators are set here: Can the location information of the bicycles available be updated at any time and whether it is convenient to find; Can the service personnel respond to user questions in a timely manner; Can the damaged vehicle be repaired in time; If the user has an accident or injury due to a shared bicycle, Whether the bike-sharing company should be held responsible; when a money dispute occurs, can it be handled and refunded in time.

\subsection{Guarantee}

Assurance refers to the knowledge, etiquette and ability of employees to express confidence and credibility, and they can get appropriate support from the company to provide better services. Four indicators are set here: whether the user's private information is protected; whether the bicycle charging is reasonable and whether the payment is safe; whether the service personnel are patient and polite; 
whether the software platform is improving and whether the quality of the bicycle is improving.

\subsection{Empathy}

Empathy refers to caring and providing personalized services to customers, thinking about problems from the perspective of users. Here are 7 indicators: whether the service personnel have the ability to differentiate services; whether the bicycle charging standards are differentiated between peak and flat peaks; whether the vehicle design meets the needs of all people; whether the operating company often actively understands the user's use status; whether the operating company Provide accurate and usable feedback channels; Whether the user's interests can be put in the first place when providing services; Whether the delivery of vehicles varies for different regions.

\section{Evaluation method of shared bicycle service quality based on the SERVQUAL model}

\subsection{Evaluation principle}

The SERVQUAL (Service Quality) model is a tool to measure service quality. Its core is: Service quality depends on the degree of difference between the service level perceived by the user and the service level expected by the user. The model is: SERVQUAL score = actual feeling score-expected score. The SERVQUAL model divides service quality into five attributes: tangibility, reliability, responsiveness, security, and empathy. Each attribute is subdivided into several questions. Through questionnaires, users can Score the expected value and actual feeling value. It is explained by 22 specific factors related to its establishment. Finally, the SQ score is obtained through questionnaire survey, customer scoring and comprehensive calculation, which indicates the final service quality level.

\subsection{Analysis of the difference between perception and expectation}

Through the perception of specific indicators and the expected difference, objectively analyse the objective performance of various specific indicators of Haro Bike. None of the 27 evaluation indicators of Harbin's bicycle service quality can meet passengers' expectations. However, in terms of core indicators such as software operation, vehicle riding experience, bicycle quality, and rental methods, users 'service quality expectations and experience values are basically the same, indicating that the use of shared bicycles almost meets the actual needs of users. There are even evaluations of shared bicycles exceeding expectations in the data.

Among the public indicators, the difference between the "vehicle scheduling is timely and whether there is a car available at any time" indicator has the highest difference. It can be seen that the number of Haro bicycles, the scheduling situation, and the unified management after the release are far from user expectations. After online investigations, it was found that Haro bicycles entered Huai'an for the first time in January 2018, with a volume of about 2,000 vehicles, and the area was small, the number was small, and there was no fixed collection point. After use, users stopped at any time. During the field investigation, it was found that there are more people riding on bicycles, but fewer idle vehicles are available. In crowded places such as urban areas, an idle bicycle is often not seen within hundreds of meters. And for a user who wants to get convenience through shared bicycles, it will greatly reduce their actual experience of shared bicycles.

Table1. Descending order of the difference of each indicator

\begin{tabular}{|c|c|}
\hline Indicator & Value \\
\hline Whether the vehicle dispatching is timely, and whether there are vehicles available at any time & -1.88 \\
\hline Is there any difference between peak and flat toll rates for bicycles & -1.84 \\
\hline Whether the cycling records and deductions are accurate & -1.82 \\
\hline Whether the service personnel can respond to user questions in a timely manner & -1.80 \\
\hline $\begin{array}{l}\text { Regarding the legal requirement that under } 12 \text { years old cannot ride a bicycle, does the operating } \\
\text { company Some measures }\end{array}$ & -1.68 \\
\hline Can the interests of users be put first when providing services & -1.66 \\
\hline $\begin{array}{l}\text { If the user has an accident or injury due to a shared bicycle, the shared bicycle Whether the company } \\
\text { should take responsibility }\end{array}$ & -1.64 \\
\hline
\end{tabular}


Does the operating company often take the initiative to understand the user's usage status

Whether users' private information is protected

Whether the delivery of vehicles varies according to different regions

In the event of a money dispute, can it be handled and refunded in time

If the user encounters a sudden failure on the way, is there a rescue mechanism?

Is it possible to update the location information of the available bicycles at any time, and is it easy to find

Whether the operating company provides accurate and usable feedback channels

Whether the bicycle charge is reasonable and whether the payment is safe

Whether the service personnel have the ability to differentiate services

Whether the brakes of the vehicle are sensitive during riding and whether it can be stopped immediately

Does the vehicle design meet the needs of all groups of people

Whether the damaged vehicle can be repaired in time

Whether the vehicle is damaged or not- 0.56

Whether the service staff is patient and polite

Whether credit free car rental and other activities are honoured

Whether the vehicle design is novel, fashionable and beautiful

Whether the vehicle is comfortable and light to ride

Whether the QR code is clear or not damaged

Whether the software platform is improving and the quality of the bicycle is improving

\section{Conclusion}

Based on the SERVQUAL model, this paper proposes an evaluation index system and evaluation method for urban shared bicycles, and carries out a case analysis in combination with Huaian Harbin bicycles.

The study found that Huaian Haro's bicycle service quality score is -1.053 points, which is a C-level service quality. Through the analysis of the average value of the difference of each indicator, the main problems found are: the amount of Haro bicycles does not meet the expectations of users; the customer service response speed. The degree is not good, and the robot customer service is not smart enough; the price of bicycles is gradually increasing, and there is no flexible charging plan and deposit standard; Children under 12 years old frequently use cars; Users are concerned about information leakage.
In view of the above problems, improvements can be considered from the following aspects: Make full use of the data brought by the shared bicycle's own positioning system, monitor the high-frequency use area of the bicycle through the background data, and combine the time period, the flow of people, the surrounding facilities and other information, To formulate a more reasonable placement area and quantity. At the same time, it can increase the investment in bicycle deployment personnel; Combine the various problems that have occurred, develop a clear and detailed automatic reply list, accurately point to the situation that the user is facing, and increase the investment in consultants; Conduct preferential activities on a regular basis to stabilize User satisfaction, while cooperating with corresponding policies, introducing new capital, formulating a flexible charging system, and introducing good faith submission, reducing or exempting part of the deposit; The user should add corresponding 
clauses when registering, and increase the prompts of the corresponding prohibitions in the operation interface.

\section{References}

1. Bentler, P., R. Bagozzi, et al. (2008) SEM using correlation or covariance matrices. Journal of Consumer Psychology, 10(2):203-252.

2. Chatterjee, S., M. H. Lubatkin, et al. (2016) Cultural differences and shareholder value in related mergers: Linking equity and human capital. Strategic Management Journal, 13(5): 319-334

3. Chircu, A. M. and R. J. Kauffman. (2016) Limits to value in electronic commerce-related IT investments. Journal of Management Information Systems, 17(2):37-63.

4. Jarvenpaa, S. L. and P. A. Todd Consumer reactions to electronic shopping on the World Wide Web [J]. International Journal of Electronic Commerce, 1(2):29-54.

5. Pateli, A. G. and G. M. Giaglis. A research framework for analysing eBusiness models. European Journal of Information Systems, 13(4):101134. 